\title{
Repeated allergen exposure changes collagen composition in airways of sensitised Brown Norway rats
}

\author{
E. Palmans, R.A. Pauwels, J.C. Kips
}

Repeated allergen exposure changes collagen composition in airways of sensitised Brown Norway rats. E. Palmans, R. A. Pauwels, J.C. Kips. (C) ERS Journals Ltd 2002. ABSTRACT: Increased or altered collagen deposition in the airway wall is one of the characteristics of airway remodelling in asthma. The mechanisms underlying this increase, and its functional consequences remain to be established further. Representative in vivo animal models might be useful in this respect.

In the present study, collagen deposition after prolonged allergen exposure was characterised in the airway wall of Brown Norway rats. Sensitised rats were repeatedly exposed to ovalbumin (OA) or phosphate-buffered saline during 2 and 12 weeks.

The deposition of collagen type I, III, IV, V and VI was not altered in animals exposed to $\mathrm{OA}$ for 2 weeks. After 12 weeks of $\mathrm{OA}$ exposure, more collagen type I was deposited in the inner and outer airway wall and more type $V$ and VI collagen was observed in the outer airway wall. At 12 weeks the number of vessels, identified via type IV collagen staining was not increased, but the total vessel area was.

In conclusion, prolonged allergen exposure in sensitised rats is associated with enhanced deposition of type I, V and VI collagens and increased vascularity. This suggests that some aspects of airway remodelling in asthma could be driven by longterm allergen exposure.

Eur Respir J 2002; 20: 280-285.

Dept of Respiratory Diseases, Ghent University Hospital, Ghent, Belgium.

Correspondence: E. Palmans, Dept of Respiratory Diseases, Ghent University Hospital, De Pintelaan 185, B-9000 Ghent, Belgium.

Fax: 3292402341

E-mail: els.palmans@hogent.be

Keywords: Airway, collagen, rat

Received: June 262001

Accepted after revision: March 132002

This study was supported by the Concerted Research Initiative of Ghent University (Grant No. GOA 98-6) and GlaxoWellcome (courtesy of M. Johnson).
Different extracellular matrix (ECM) proteins have been identified throughout the airway wall. The basement membrane (BM) underneath the airway epithelium consists of type IV collagen, proteoglycans, laminin, and fibronectin [1]. Beneath this BM the ECM of normal airways is composed of collagens type I, III and V, elastins, proteoglycans, fibronectin and laminins. The major fibrillar components of the matrix, type I and III collagen and elastin, are the primary determinants of the mechanical properties of airways [2].

The asthmatic airway wall displays structural changes including sub-basement membrane thickening, altered submucosal ECM deposition and neovascularisation $[1,3-6]$. The thickened layer beneath the BM comprises predominantly types I, III, and V collagen together with fibronectin and tenascin $[1,7]$. Moreover, increased deposition of collagen type III, V and VI has also been observed in the bronchial submucosa in chronic asthmatics [3, 4]. Others have observed the presence of increased amounts of fragmented elastin fibres under the epithelium [5]. Both the pathogenesis and the functional consequences of these ECM changes in asthmatic airways remain uncertain.

An appropriate in vivo animal model could provide useful information in this respect. The current authors have previously reported that prolonged allergen exposure induced structural changes in the airways of sensitised Brown Norway (BN) rats [8]. In these animals exposure to allergen for up to 3 months resulted in an increased amount of collagen and fibronectin in the large airways [8]. The increased collagen deposition is further characterised here by evaluating the amount of different collagen types in the airway wall of sensitised rats exposed to allergen for 14 days and 3 months.

\section{Material and methods}

\section{Sensitisation and exposure of animals}

Male BN rats (Harlan CPB, Zeist, the Netherlands) were sensitised and exposed to allergen as described previously [8]. In brief, all animals were sensitised by an intraperitoneal (i.p.) injection of $1 \mathrm{mg}$ ovalbumin (OA) (Sigma Chemical Co., Poole, UK) and $200 \mu \mathrm{g}$ $\mathrm{Al}(\mathrm{OH})_{3}$ on day 0 . One group of animals $(\mathrm{n}=5)$ was boosted with $1 \mathrm{mg} \mathrm{OA}$ and $200 \mu \mathrm{g} \mathrm{Al}(\mathrm{OH})_{3}$ i.p. on day 7 and exposed from day $14-28$ to aerosolised 1\% OA thrice weekly (2 weeks). A second group of animals $(\mathrm{n}=5)$ was similarly exposed to aerosolised $1 \%$ OA from days 14-98 (12 weeks). Control animals $\left(\mathrm{n}=5 \cdot\right.$ group $\left.^{-1}\right)$ were exposed to sterile phosphatebuffered saline (PBS). 


\section{Lung histology}

Rats were anaesthetised $\left(60 \mathrm{mg} \cdot \mathrm{kg}^{-1}\right.$ pentobarbital (Sanofi, Libourne, France) i.p.) $24 \mathrm{~h}$ after the last aerosol exposure, and the trachea was cannulated. Following lavage, lungs were fixed with 4\% paraformaldehyde via the tracheal canulla. Lung sections were processed for immunohistochemistry and for Congo Red staining. The total number of peribronchial eosinophils was determined on Congo Red stained sections [8].

\section{Immunohistochemical staining of type I, III, IV, V and VI collagen}

Type I collagen was stained with a polyclonal goat anti-type I collagen in lung tissue embedded in Technovit 8100 (Heraeus Kulzer GmbH, Wehrheim, Germany) according to the manufacturers instructions. The other collagen types were detected in paraffin embedded tissue. Sections of $2 \mu \mathrm{m}$ were pretreated with hydrogen peroxide $\left(\mathrm{H}_{2} \mathrm{O}_{2}\right)$ to block endogenous peroxidase activity and $0.1 \%$ trypsin (Sigma Chemical) in $0.1 \% \mathrm{CaCl}_{2}(\mathrm{pH} 7.8)$ at $37^{\circ} \mathrm{C}$. The sections were rinsed in distilled water and subsequently incubated with normal rabbit serum (NRS) for $1 \mathrm{~h}$ and with the primary polyclonal antibody (Southern Biotechnology Associates Inc., Birmingham, AL, USA) diluted in 1\% NRS as follows: goat anti-type I collagen (1:20), goat anti-type III collagen (1:100), goat anti-type IV collagen (1:50), goat anti-type V collagen (1:50) and goat anti-type VI collagen (1:300). The sections were washed and incubated with biotinylated rabbit antigoat immunoglobulin-G (DAKO A/S, Glostrup, Denmark) diluted 1:200. The primary antibody-secondary antibody complex was detected with the streptavidinbiotinylated horseradish peroxidase complex (Nycomed Amersham Plc, Buckinghamshire, UK) diluted 1:200. Peroxidase activity was developed in diaminobenzidine (DAB) (DAKO), giving a brown reaction product. Sections were counterstained with haematoxylin. Rat skin was used as a positive control for the different collagen types. The primary antibody was substituted with normal goat serum as a negative control.

\section{Quantification of collagen deposition}

The deposition of the different types of collagen was examined by light microscopy and quantitative measurements were performed using a computerised image analysis system (Leica Q500MC; Leica Cambridge Ltd, Cambridge, UK) as described previously [8]. For each experimental group three lung sections per animal were examined. The amount of specific collagen deposited in the different compartments of the airway wall was measured in all large airways (length of $\mathrm{BM}(\mathrm{Pbm})>2 \mathrm{~mm})$ cut in reasonable cross sections (defined by a ratio of minimal to maximal internal diameter $<0.5$ ). The different compartments of the airway wall are: the total wall, defined by the BM and the outer adventitia; the inner wall, defined by the BM and the outer boundary of the smooth muscle; and the outer wall, defined by the outer boundary of the smooth muscle and the outer adventitia. The borders of these compartments were marked manually and the amount of specific collagen deposited in these regions was analysed by software.

\section{Measurement of vessel area}

Vessels were identified by the staining of their BM with type IV collagen. With the image analysis system (Leica Q500MC) the vessel area in the airway wall of large airways was measured on the sections used for type IV collagen quantification. The vessel area is defined by all the structures internal to the vessel BM and was calculated by Qwin software (Leica Cambridge Ltd). The total amount of vessels in the total airway wall were counted.

\section{Data analysis}

Reported values are expressed as mean \pm SEM. The data on collagen deposition, vessel area and vessel number in all sections in one experimental group were pooled together. Each quantification was performed in a mean of 16 large airways (range 12-25) per experimental group. Values of the area of collagen deposition in total, inner and outer airway wall were normalised to $\mathrm{Pbm}$. The vessel area was normalised to the total wall area and the number of vessels were divided by the total wall area to obtain the amount of vessels $\cdot \mathrm{mm}^{-2}$. The frequency distribution of $\mathrm{Pbm}$ for the PBS and OA experimental groups was compared using the Kolmogorov-Smirnov test to ensure the comparison of a similar range of airway sizes. Mean values of the total, inner and outer airway wall, the vessel area and the amount of vessels $\cdot \mathrm{mm}^{-1}$ were compared between the OA and PBS-exposed animals in one experiment by an unpaired t-test. Spearman's rank correlation coefficient was used to examine the correlation between the amount of collagen deposited in the airway wall and the number of peribronchial eosinophils. p-Values $<0.05$ were regarded as significant.

\section{Results}

\section{Distribution of different collagen types}

Type I collagen was clearly present in the total airway wall and formed bundles parallel with the airway epithelium (fig. 1a and b). Type III and type VI collagen were ubiquitous in the airway mucosa and adventitia and their deposition pattern was similar (fig. 1c, g and h). Type V collagen was predominantly deposited in the outer airway wall close to the airway smooth muscle cells and the blood vessels (fig. 1f). Type IV collagen was found in the basement membrane beneath the epithelium and surrounding blood vessels, and within smooth muscle bundles (fig. 1e).

The distribution pattern of the different types of collagen was similar in control animals and 

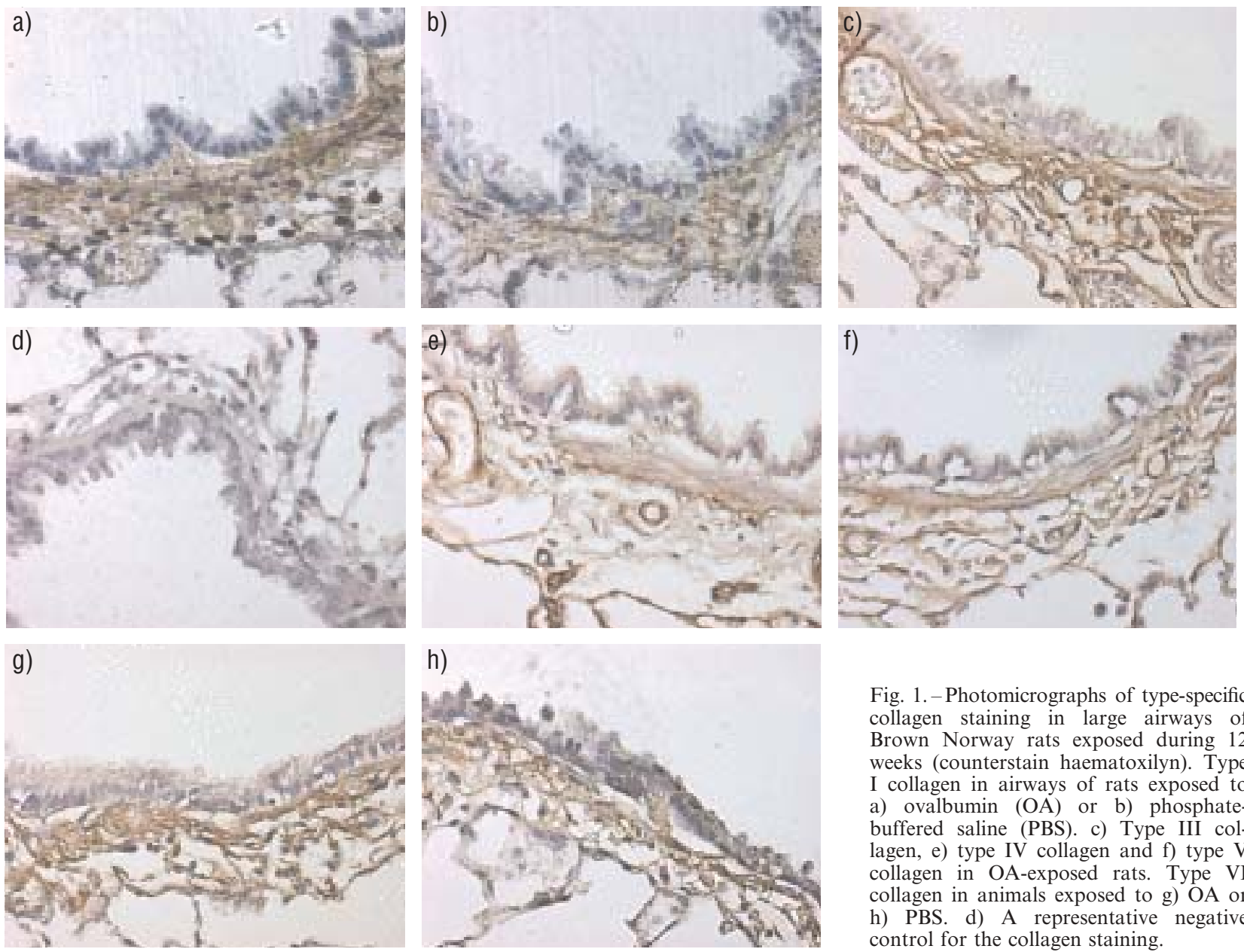

Fig. 1.-Photomicrographs of type-specific collagen staining in large airways of Brown Norway rats exposed during 12 weeks (counterstain haematoxilyn). Type I collagen in airways of rats exposed to a) ovalbumin (OA) or b) phosphatebuffered saline (PBS). c) Type III collagen, e) type IV collagen and f) type V collagen in OA-exposed rats. Type VI collagen in animals exposed to g) OA or h) PBS. d) A representative negative control for the collagen staining.

allergen-exposed animals independent of the duration of the exposure (fig. 1).

\section{Quantification of type I, III, V and VI collagen deposition}

The amount of the collagen subtypes deposited in the total, inner and outer airway wall of large airways did not differ between sensitised $\mathrm{BN}$ rats exposed to PBS or OA during 2 weeks (data not shown).

Quantitative changes in collagen types were observed in rats exposed to allergen during 12 weeks. Type I and type VI collagen were increased in the total airway wall $(\mathrm{p}<0.001$ versus PBS-exposed animals) (fig. 2a and c). The increase in type I collagen was observed in the inner $(\mathrm{p}<0.01)$ as well as in the outer airway wall $(\mathrm{p}<0.001)($ fig. $2 \mathrm{a})$ and the increase in type VI collagen was found mainly in the outer airway wall $(p<0.001)$ (fig. $2 c)$. Although the amount of type $\mathrm{V}$ collagen in the total airway wall of OA and PBS-exposed rats did not differ, more type V collagen was deposited in the outer airway wall of allergen exposed animals $(\mathrm{p}<0.05$ versus $\mathrm{PBS})$ (fig. $2 \mathrm{~b})$. No difference was observed in the deposition of type III collagen between OA and PBS-exposed rats $\left(12.56 \pm 1.24\right.$ versus $\left.13.93 \pm 2.33 \mu \mathrm{m}^{2} \cdot \mu \mathrm{m} \cdot \mathrm{BM}^{-1}\right)$.
The amount of type I collagen present in the airway wall correlated with the number of peribronchial eosinophils in rats exposed during 12 weeks (rank correlation coefficient $\left.\left(\mathrm{r}_{\mathrm{s}}\right)=0.72, \mathrm{p}<0.05\right) \quad$ (fig. 3). Deposition of type VI collagen was also related to the number of eosinophils in the airway wall $\left(r_{s}=0.86\right.$, $\mathrm{p}<0.01$ ) (data not shown). The amount of type $\mathrm{V}$ collagen in the airway wall did not correlate with the number of eosinophils.

\section{Basement membrane type IV collagen}

No differences were observed in the amount of type IV collagen deposited in the airway wall of rats exposed to $\mathrm{OA}$ during 2 and 12 weeks $(1.51 \pm 0.21$ versus $1.33 \pm 0.4 \mu \mathrm{m}^{2} \cdot \mu \mathrm{m} \cdot \mathrm{BM}^{-1}$ in $\mathrm{PBS}$-exposed animals). Also, no difference was seen in the amount of vessels per area of the bronchial wall. After 2 weeks, the airways of OA-exposed animals contained 265 \pm 27 vessels $\cdot \mathrm{mm}^{-2}$ compared to $332 \pm 20$ vessels $\cdot \mathrm{mm}^{-2}$ in PBS-exposed rats. After 12 weeks $318 \pm 13$ vessels $\cdot \mathrm{mm}^{-2}$ were counted in the OA group compared to $288 \pm 31$ vessels $\cdot \mathrm{mm}^{-2}$ in the PBS group. The vessel area normalised to the total wall area was increased in animals exposed to OA during 12 weeks (fig. 4) compared to PBS-exposed animals $(\mathrm{p}<0.05)$. 

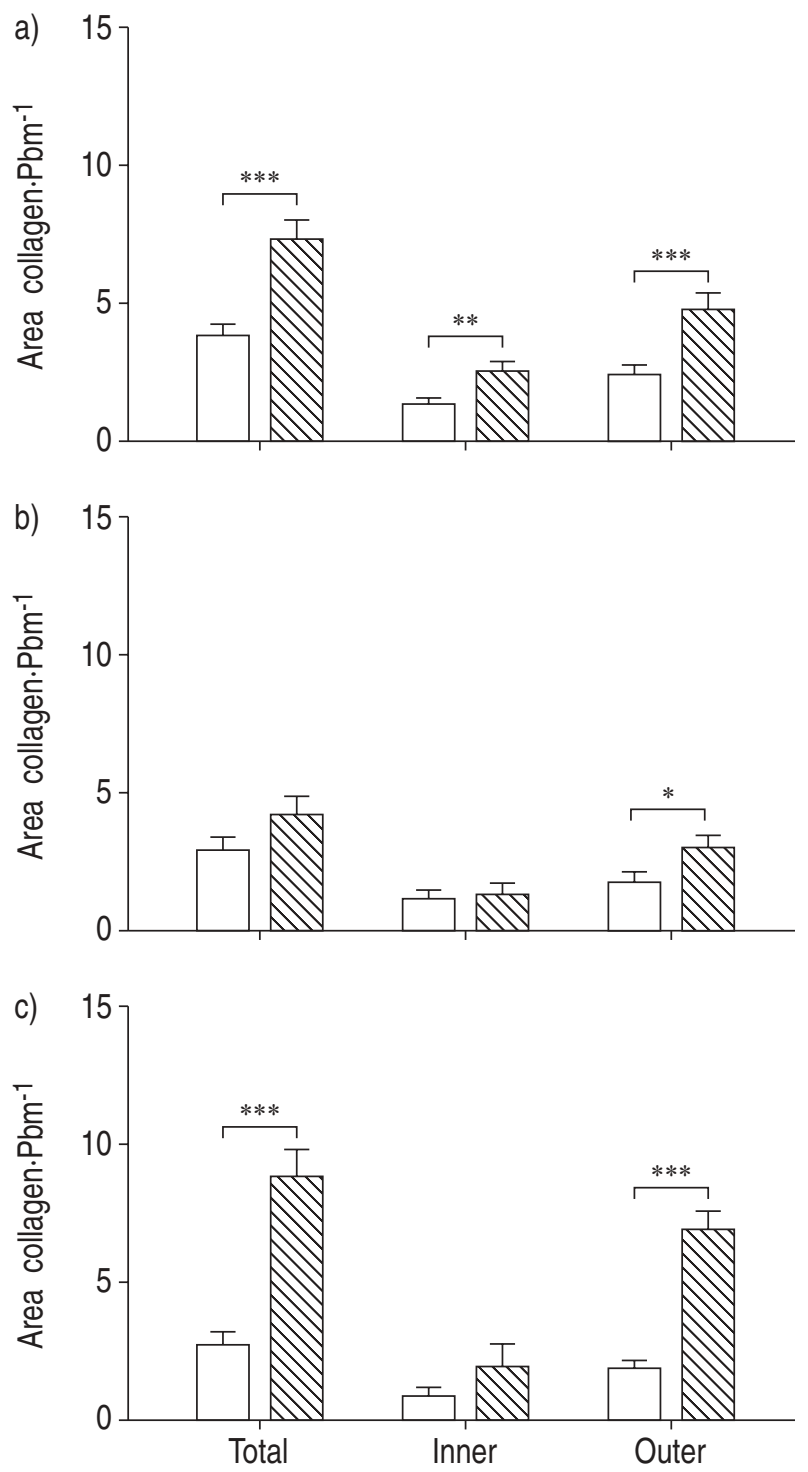

Fig. 2. - Deposition of type I, V and VI collagen in the total, inner and outer airway wall of sensitised rats exposed to phosphatebuffered saline $(\square)$ or ovalbumin $(\mathbb{\mathbb { Q }})$ during 12 weeks. $*$ : p $<0.05$; **: $\mathrm{p}<0.01 ; * * *: \mathrm{p}<0.001$.

\section{Discussion}

The purpose of this study was to characterise changes in collagen deposition in the large airway walls of sensitised $\mathrm{BN}$ rats repeatedly exposed to allergen [8]. The results indicate that these airways contained increased amounts of collagen types I, V and VI after 12 weeks of allergen exposure. An increase of all three collagen types was observed in the outer airway wall; collagen type I was also deposited in the inner wall. No differences were observed in the deposition of type III and type IV collagen. Vascular perfusion was increased after 12 weeks of OA exposure.

Two aspects of changes in the collagen composition in the airway wall, that have been linked to chronic asthma, are the thickened lamina reticularis and increased deposition of collagen in the submucosa $[3,4]$. The information obtained from endobronchial

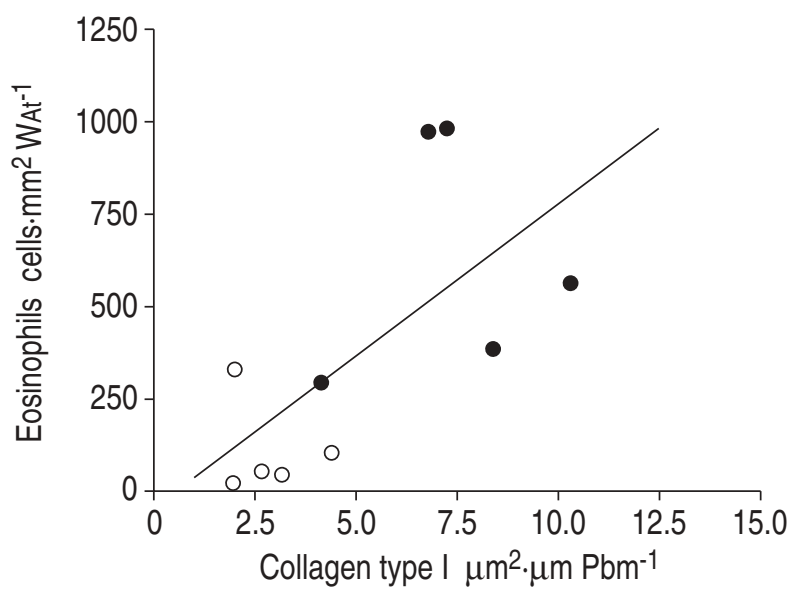

Fig. 3.-Correlation between the amount of type I collagen deposited in the airway wall and the number of peribronchial eosinophils in Brown Norway rats. $\bigcirc$ : rats exposed to phosphatebuffered saline; 0 : rats exposed to ovalbumin. Rank correlation coefficient $\left(\mathrm{r}_{\mathrm{s}}\right)=0.721, \mathrm{p}<0.05$. WAt: total wall area.

biopsy studies relates mainly to the composition of the area beneath the basal lamina. In the asthmatic airway the reticular layer represents an area of irregularly distributed fibrosis. This collagenous matrix band beneath the BM contains increased amounts of the fibrillar collagens type I, III and V, but also of fibronectin and tenascin $[1,3,7]$. In the present model the increase in type I collagen in this area is most prominent. Although the thickness of the lamina reticularis is increased in bronchial biopsies from asthmatic when compared to normal subjects $[1,9]$ the significance of this subepithelial fibrosis remains unclear. In some studies, no relation was found between the thickening and the severity, duration or aetiology of asthma [9-11]. Others confirmed thickening of the matrix band beneath the BM in atopic, but not in nonatopic asthma [12], and reported a correlation with the severity but not with the duration of asthma [13, 14].

Far less data are available on the distribution of collagen in the submucosa and the adventitial area.

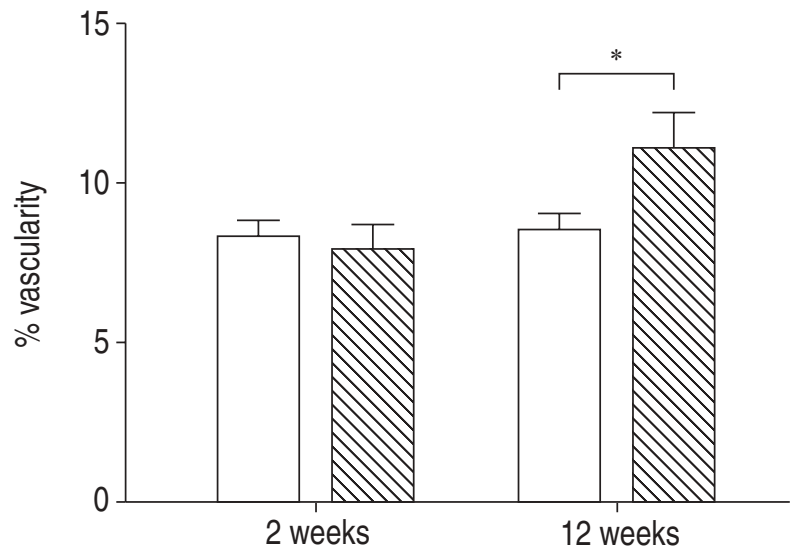

Fig. 4. - The effect of phosphate-buffered saline ( $\square$ ) or ovalbumin (\$) exposure during 2 weeks and 12 weeks on the vascularity in large airways. *: $\mathrm{p}<0.05$. 
WILSON and Li [3] observed increased amounts of type III and type V collagen in the submucosa of asthmatic airways and LeE et al. [4] found an increase in type VI collagen in the submucosa of asthmatics. These findings have invariably not been confirmed, as others did not observe increased amounts of total collagen nor increased deposition of type I and III collagen in asthmatic subjects [10]. In the present study, repeated allergen challenge was shown to induce increased deposition of collagen I, V and VI in the outer airway wall. This area corresponds to the adventitial tissue between the cartilage and/or the smooth muscle layer. In normal airways, the adventitia consists mainly of fibrous tissue and blood vessels, but to the best of the current authors knowledge, data on collagen deposition in the adventitia of the asthmatic airways are not available. It is therefore unclear whether subepithelial fibrosis serves as a marker of changes, such as ECM protein deposition throughout the submucosa and the adventitia, as has been suggested [15]. Therefore, the current authors cannot ascertain that the changes observed in this study are consistent with those in human asthma.

Data on vascular changes within the airway wall of asthmatics are also sparse. Recent reports illustrate an increase of vessels and vascularity in the airway wall of asthmatics [16-18]. In patients with fatal asthma, the number of large blood vessels was increased, compared to control subjects, in large but not in smaller airways [19]. Similarly, the total area occupied by the blood vessels in the airway submucosa was not different between asthmatics and control subjects [19]. In the present model, blood vessels were identified by immunostaining for collagen type IV, which is the major collagen subtype present in the vascular BM. Using this approach, an increase in the total amount of vessels $\cdot \mathrm{mm}^{-2}$ could not be identified. However, in line with the data by LI and WILSON [6], an increase in the total area of the mucosa occupied by blood vessels was found.

The mechanisms underlying the allergen-induced increased collagen deposition in this model remain to be further elucidated. Collagen formation is part of the tissue repair response to injury, in an attempt to regenerate tissue. When this repair process is dysregulated and prolonged, it may result in progressive fibrosis with its specific changes [20]. Different types of collagen seem to be produced at various stages in the regeneration process. The production of ECM in rat airways repeatedly exposed to allergen, resembles the process of tissue regeneration in which fibronectin precedes collagen deposition forming a provisional ECM [21]. Previously, the current authors have shown that changes in fibronectin deposition start after 2 weeks, but that the increase in collagen content and composition of the airway wall is observed only after a longer exposure period [22]. Moreover, collagen type I and $\mathrm{V}$ are usually considered to reflect fully established fibrotic lesions, occurring at a later stage during the regeneration process $[23,24]$. The main sources of collagen are probably fibroblasts and/or myofibroblasts, responding to a wide range of stimuli, including various growth factors such as transforming growth factor- $\beta$, insulin-like growth factor or platelet-derived growth factor $[25,26]$. These factors can, in turn, be released from different cell types, epithelial cells and eosinophils in particular [26-28]. Of note is the positive correlation between the amount of collagen type I and VI and the number of eosinophils in the airway wall of $\mathrm{BN}$ rats exposed during 12 weeks, found in the present study. Whether the eosinophils express these growth factors is so far unknown.

The increased and altered collagen deposition in the airway wall observed in the current study could have important functional consequences. Mathematical models link airway remodelling to airway hyperresponsiveness (AHR) [29]. Changes in the composition of the airway connective tissue can influence smooth muscle shortening [30], and depending on the extent and location of the altered ECM deposition, these might protect against instead of enhancing airway responsiveness to bronchoconstrictor stimuli [31-33]. In the initial study, the current authors observed that the loss of AHR in rats exposed to OA during 12 weeks was associated with an increase in total collagen in the total airway wall and fibronectin in the outer airway wall [22]. The authors suggested that tighter adhesion between lung parenchyma and the outer airway wall could influence the mechanical properties of the airways [22]. Type VI collagen microfibrils are known to form fibrillar connections between other ECM components [34]. These fibres might thus function as a mechanical link between interstitial collagen fibrils and other matrix structures including basement membranes and elastin microfibrils [35]. Type I collagen forms a relatively rigid linear fibrillar network and could also increase the stiffness of the airway wall [36]. The increase of both collagen type I and VI in the OA-exposed rats might therefore increase rigidity of the airway wall and thus oppose against instead of induce AHR.

These observations stress the importance of including all structural changes that occur throughout the airway wall, when evaluating the functional role of remodelling. To date, in man, this analysis has been limited to subepithelial collagen deposition, relating the extent of collagen type I and III deposition to an increase as opposed to a decrease in airway responsiveness. The causal relationship between both however is not known. The present model cautions against relating one single aspect of the structural alterations to airway function, without taking into account the potential contribution of co-existing alterations.

In conclusion, the increased collagen type I, VI and $\mathrm{V}$ deposition in the airway wall of sensitised rats exposed to allergen during 12 weeks might result from repeatedly induced tissue repair processes in response to the persistent presence of inflammatory cells. The different collagens may increase the rigidity and stiffness of the airway wall and thus prevent airway hyperresponsiveness.

\footnotetext{
Acknowledgements. The authors wish to thank A. Neessen and I. De Borle for technical assistance.
} 


\section{References}

1. Roche WR, Beasley R, Williams JH, Holgate ST Subepithelial fibrosis in the bronchi of asthmatics. Lancet 1989; 1: 520-524.

2. Campa JS, Harrison NK, Laurent GJ. Regulation of matrix production in the airways. In: Jolles G, Karlsson JA, Taylor J, eds. T-Lymphocyte and inflammatory cell research in asthma. London, Academic Press Ltd, 1993: pp. 221-239.

3. Wilson JW, Li X. The measurement of reticular basement membrane and submucosal collagen in the asthmatic airway. Clin Exp Allergy 1997; 27: 363-371.

4. Lee K, Li X, Carroll N, James A, Wilson JW. Subepithelial and submucosal collagen in fatal asthma. Am J Respir Crit Care Med 1999; 157: A840.

5. Bousquet J, Lacoste JY, Chanez P, Vic P, Godard P, Michel FB. Bronchial elastic fibers in normal subjects and asthmatic patients. Am J Respir Crit Care Med 1996; 153: 1648-1654.

6. Li X, Wilson JW. Increased vascularity of the bronchial mucosa in mild asthma. Am J Respir Crit Care Med 1997; 156: 229-233.

7. Laitinen A, Altraja A, Kampe M, Linden M, Virtanen I, Laitinen L. Tenascin is increased in airway basement membrane of asthmatics and decreased by an inhaled steroid. Am J Respir Crit Care Med 1997; 156: 951-958.

8. Palmans E, Kips JC, Pauwels RA. Prolonged allergen exposure induces structural airway changes in sensitized rats. Am J Respir Crit Care Med 2000; 161: 627635.

9. Jeffery PK, Wardlaw AJ, Nelson FC, Collins JV, Kay AB. Bronchial biopsies in asthma. An ultrastructural, quantitative study and correlation with hyperreactivity. Am Rev Respir Dis 1989; 140: 1745-1753.

10. Chu HW, Halliday JL, Martin RJ, Leung DY, Szefler SJ, Wenzel SE. Collagen deposition in large airways may not differentiate severe asthma from milder forms of the disease. Am J Respir Crit Care Med 1998; 158: 1936-1944.

11. Milanese M, Crimi E, Scordamaglia A, et al. On the functional consequences of bronchial basement membrane thickening. J Appl Physiol 2001; 91: 1035-1040.

12. Amin K, Ludviksdottir D, Janson C, et al. Inflammation and structural changes in the airways of patients with atopic and nonatopic asthma. Am J Respir Crit Care Med 2000; 162: 2295-2301.

13. Chetta A, Foresi A, Del Donno M, Bertorelli G, Pesci A, Olivieri D. Airways remodeling is a distinctive feature of asthma and is related to severity of disease. Chest 1997; 111: 852-857.

14. Boulet LP, Laviolette M, Turcotte H, et al. Bronchial subepithelial fibrosis correlates with airway responsiveness to methacholine. Chest 1997; 112: 45-52.

15. Bradding P, Redington AE, Holgate ST. Airway wall remodelling in the pathogenesis of asthma: cytokine expression in the airways. In: Steward AG, ed. Airway wall remodelling in asthma. Boca Raton, USA, CRC Press, Inc., 1997; pp. 29-63.

16. Skalli O, Ropraz P, Trzeciak A, Benzonana G, Gillessen D, Gabbiani G. A monoclonal antibody against alpha-smooth muscle actin: a new probe for smooth muscle differentiation. J Cell Biol 1986; 103 : 2787-2796.

17. Kuhn C3, Boldt J, King TE Jr, Crouch E, Vartio T, McDonald JA. An immunohistochemical study of architectural remodeling and connective tissue synthesis in pulmonary fibrosis. Am Rev Respir Dis 1989; 140: 1693-1703.

18. Orsida BE, Ward C, Li X, et al. Effect of a long-acting $\beta_{2}$-agonist over three months on airway wall vascular remodeling in asthma. Am J Respir Crit Care Med 2001; 164: 117-121.

19. Carroll NG, Cooke C, James AL. Bronchial blood vessel dimensions in asthma. Am J Respir Crit Care Med 1997; 155: 689-695.

20. Busse W, Elias J, Sheppard D, BanksSchlegel S. Airway remodeling and repair. Am J Respir Crit Care Med 1999; 160: 1035-1042.

21. Welch MP, Odland GF, Clark RA. Temporal relationships of F-actin bundle formation, collagen and fibronectin matrix assembly, and fibronectin receptor expression to wound contraction. $J$ Cell Biol 1990; 110: 133-145.

22. Palmans E, Kips JC, Pauwels RA. Prolonged allergen exposure induces structural airway changes in sensitized rats. Am J Respir Crit Care Med 2000; 161: 627-635.

23. McAnulty RJ, Laurent GJ. Collagen and its regulation in pulmonary fibrosis. In: Phan SH, Thrall RS, eds. Pulmonary fibrosis. New York, NY, Marcel Dekker, Inc., 1995; pp. 135-171.

24. Martin GR, Timple R, Müller PK, Kühn K. The genetically distinct collagens. IBS 1985: 285-287.

25. Hoshino M, Nakamura Y, Sim JJ. Expression of growth factors and remodelling of the airway wall in bronchial asthma. Thorax 1998; 53: 21-27.

26. Zhang S, Smartt H, Holgate ST, Roche WR. Growth factors secreted by bronchial epithelial cells control myofibroblast proliferation: an in vitro co-culture model of airway remodeling in asthma. Lab Invest 1999; 79: 395-405.

27. Vignola AM, Chanez P, Chiappara G, et al. Transforming growth factor- $\beta$ expression in mucosal biopsies in asthma and chronic bronchitis. Am J Respir Crit Care Med 1997; 156: 591-599.

28. Ohno I, Nitta Y, Yamauchi K, et al. Eosinophils as a potential source of platelet-derived growth factor B-chain (PDGF-B) in nasal polyposis and bronchial asthma. Am J Respir Cell Mol Biol 1995; 13: 639-647.

29. James AL, Pare PD, Hogg JC. The mechanisms of airway narrowing in asthma. Am Rev Respir Dis 1989; 139: 242-246.

30. McFawn PK, Mitchell HW. Bronchial compliance and wall structure during development of the immature human and pig lung. Eur Respir J 1997; 10: 27-34.

31. Lambert RK, Codd SL, Alley MR, Pack RJ. Physical determinants of bronchial mucosal folding. $J$ Appl Physiol 1994; 77: 1206-1216.

32. Seow CY, Wang L, Pare PD. Airway narrowing and internal structural constraints. J Appl Physiol 2000; 88: 527-533.

33. Milanese M, Crimi E, Scordamaglia A, et al. On the functional consequences of bronchial basement membrane thickening. J Appl Physiol 2001; 91: 1035-1040.

34. Davidson JM. Biochemistry and turnover of lung interstitium. Eur Respir J 1990; 3: 1048-1068.

35. Keene DR, Engvall E, Glanville RW. Ultrastructure of type VI collagen in human skin and cartilage suggests an anchoring function for this filamentous network. J Cell Biol 1988; 107: 1995-2006.

36. Raghow R. The role of extracellular matrix in postinflammatory wound healing and fibrosis. FASEB $J$ 1994; 8: 823-831. 